\title{
SOME OPEN PROBLEMS IN HIGHER DIMENSIONAL COMPLEX ANALYSIS AND COMPLEX DYNAMICS
}

\author{
John ERIK FornÆsS* AND Nessim Sibony
}

Abstract

We present a collection of problems in complex analysis and complex dynamics in several variables.

\section{Contents}

1. Introduction 529

2. Complex dynamics problem list 530

2.1. Discrete dynamics 530

2.1.1. Fatou sets, stable sets $\quad 530$

2.1.2. Julia sets, currents $\quad 532$

2.2. Continuous dynamics 535

3. Several domplex variable problem list 536

3.1. $\bar{\partial} \quad 536$

3.2. Levi problem 538

3.3. Holomorphic mappings 539

References $\quad 540$

\section{Introduction}

We present here a collection of problems in complex analysis and complex dynamics in several variables. The list contains some old questions which are well known and some new ones. There is no pretention to be exhaustive and the leading line was just that one of us was interested recently in these problems. The questions are of various nature, some would lead to a breakthrough and require new ideas, others are reasonably easy. We thank Bedford, Berndtsson, Burns, Henkin,

2000 Mathematics Subject Classification. 32D, 32F05, 32F20, 32H40, 32H50, 53C65, $58 \mathrm{~F} 05,58 \mathrm{~F} 11,58 \mathrm{~F} 18$.

Key words. Fatou set, biholomorphic map, Siegel domain, dimension of measures, symplectic maps, $\bar{\partial}$-equation, Levi problem, holomorphic mappings.

*The first author was supported by an NSF grant. 
Ohsawa, Pinchuk, who have proposed some of the questions. We also thank Diederich, Ebenfeldt, Jonsson, Slapar and Winkelmann for comments and suggestions on the original version and Siu for providing the solution to one of the problems.

\section{Complex dynamics problem list}

We divide the questions into discrete and continuous ones. The discrete questions are further divided in two sets depending on whether the stable or chaotic features are most dominant.

\subsection{Discrete dynamics.}

\subsubsection{Fatou sets, stable sets.}

Question 2.1. Let $\mathcal{F}$ be the family of holomorphic endomorphisms of $\mathbb{P}^{k}$ of degree $d \geq 2$ with infinitely many sinks. (We call the basin of attraction of an attracting periodic point a sink.) Can $\mathcal{F}$ have interior or have positive measure? When $k=1$ it is a result of Fatou $[\mathbf{C G}]$ that there are finitely many sinks. (One needs a critical point in each basin.) In more variables, using the Newhouse phenomenon (persistence of homoclinic tangencies), Gavosto and Buzzard constructed infinitely many sinks. The question is how frequently this happens. See [Ga] and $[\mathbf{B u}]$.

Question 2.2. Can maps on $\mathbb{P}^{k}, k \geq 2$ have wandering Fatou components? Recall that a Fatou component $\Omega$ is wandering for $f$ if $\left\{f^{n}(\Omega)\right\}_{n \geq 0}$ are two by two disjoint. When $k=1$, Sullivan, using the Ahlfors-Bers Theorem proved that there are no wandering components $[\mathbf{C G}]$. The tool used is not available in several variables. Maybe the first step is to find a new proof in one variable.

A domain $U \subset \mathbb{C}^{k}$ is a Fatou-Bieberbach domain if it is biholomorphic to $\mathbb{C}^{k}$ but $\bar{U} \neq \mathbb{C}^{k}$.

Question 2.3. Can Fatou-Bieberbach domains for Hénon maps have $\mathcal{C}^{\infty}$ boundary? Recall that a Hénon map in $\mathbb{C}^{2}$ is a biholomorphism $f$ of the following form:

$$
f(z, w)=(p(z)+a w, b z), \quad a b \neq 0,
$$

with $p$ a polynomial of degree $d \geq 2$. Assuming that 0 is an attracting fixed point it is known that $\Omega:=\left\{q ; f^{n}(q) \rightarrow p\right\}$ is biholomorphic to $\mathbb{C}^{2}$, but $\bar{\Omega} \neq \mathbb{C}^{2}$. The question is about the smoothness of $\partial \Omega$.

It is known that there are domains $U \subset \mathbb{C}^{2}, \bar{U} \neq \mathbb{C}^{2}, U$ biholomorphic to $\mathbb{C}^{2}$ and $U$ has smooth boundary $[\mathbf{S t}]$. 
Question 2.4. Let $f$ be a generalized Hénon map on $\mathbb{C}^{2}$. Let

$$
K^{ \pm}=\left\{(z, w) ;\left\{f^{ \pm n}\right\}_{n \geq 0} \text { is a bounded sequence }\right\},
$$

$J^{ \pm}=\partial K^{ \pm}$. There is a unique positive closed current $T^{ \pm}$of norm 1 supported on $K^{ \pm}$[FS10]. The measure $\mu=T^{+} \wedge T^{-}$has been studied intensely $[\mathbf{B L S}]$. The nonwandering set $\Omega(f)$ for $f$ is contained in $K:=$ $K^{+} \cap K^{-}$. What are the relations between $\Omega(f), J:=J^{+} \cap J^{-}$and $J^{*}=S_{\mu}$, the support of $\mu$ ? When is $f$ hyperbolic on $\Omega(f)$ ? There are a few cases known, perturbations of $\left(z^{d}+c, 0\right),[\mathbf{F S 3}],[\mathbf{H O}]$, more recently Hruska $[\mathbf{H}]$ has computer assisted examples of hyperbolic maps.

Question 2.5. Let $M^{k}$ be a complex manifold. Assume that $M=$ $\cup \phi_{n}(B), \phi_{n}: B \rightarrow M, 1-1$ and $\phi_{n+1}(B) \supset \phi_{n}(B), B$ is the unit ball. Assume that the Kobayashi metric $K_{M} \equiv 0$. Is $M=\mathbb{C}^{k}$ ? (False when $k \geq 3$, open when $k=2$ [FS11].)

An example of this situation is a question raised by Bedford: Let $F: \mathbb{C}^{n} \rightarrow \mathbb{C}^{n}$ be a biholomorphism, $K$ a hyperbolic saddle. Assume that the stable dimension of $K$ is $k$. For $p \in K$, is $W^{s}(p)$ biholomorphic to $\mathbb{C}^{k}$ ? This is true for periodic saddle points. It is easy to show that the Kobayashi metric vanishes on all stable leaves. See [JV] for results on generic stable manifolds.

Question 2.6. Is any "long" $\mathbb{C}^{2}$ biholomorphic to $\mathbb{C}^{2}$ ? (A complex manifold is a long $\mathbb{C}^{2}$ if it is a union of proper subsets which are biholomorphic to $\mathbb{C}^{2}$.)

Question 2.7 (M. Herman). Symplectic question: $H=(f(z)-w, z)$, $f$ entire. Find $f$ such that $f$ has a Siegel domain at $0, f(0)=0$. The problem is that the eigenvalues of $H^{\prime}(0)$ are $e^{ \pm i \theta}$ so we have resonances. Formally one gets infinitely many compatibility conditions. Recall that a Siegel domain is a domain where a subsequence of iterates converges to the identity.

Question 2.8. Can a Siegel domain for a Hénon map or, more generally, a biholomorphism of $\mathbb{C}^{2}$ have strongly pseudoconvex boundary? In one variable, R. Pérez-Marco has constructed Siegel discs with smooth boundary $[\mathbf{P M}]$.

Question 2.9. Classify periodic Fatou components for an endomorphism of $\mathbb{P}^{k}\left[\right.$ FS6]. Let $f: \mathbb{P}^{k} \rightarrow \mathbb{P}^{k}$ be an endomorphism. Assume that $U$ is a Fatou component such that $f(U)=U$. Assume that $\left(f^{n}\right)(z) \rightarrow \partial U$. Does there exist a parabolic fixed point on $\partial U$ ? See $[\mathbf{J L}]$. When $k=1$ this was done by Fatou $[\mathbf{C G}]$. 
Question 2.10. Classify those Reinhardt domains which are biholomorphic to Siegel domains, associated to an automorphism of $\mathbb{C}^{k}$ or to an endomorphism of $\mathbb{P}^{k}$.

Question 2.11. Suppose that the boundary of a Fatou component of a Hénon map has a smooth piece. What can be said about the whole boundary? In one variable the whole boundary must be smooth.

Question 2.12 (The kicked rotor; infinite dimensional complex dynamics). The kicked rotor describes a particle on a circular orbit subject to a periodic kick. In quantum mechanics it describes an elementary particle in an atom subject to a periodically pulsed electric field.

Classical description: If angular momentum is $p$, mass is $m, K$ is the kick strength, $T$ is the time between kicks, then $R(\theta, p)=\left(\theta+\frac{p T}{m}, p+\right.$ $\left.K \sin \left(\theta+\frac{p T}{m}\right)\right)$ describes the change in coordinates from just after one kick till just after the next kick. (This is also called the Standard Map.) Prove that if $k=\frac{K m}{T}>1$ then almost all orbits are unbounded.

Quantum description: If $\psi=\sum_{n \in \mathbb{Z}} c_{n} e^{i n \theta}$ is an $L^{2}$ function on $[0,2 \pi]$ describing the state of the particle after one kick, then after the next kick, the state is $R(\psi)=e^{-i \frac{K \cos \theta}{\hbar}} \sum_{n} e^{-\frac{\hbar i n^{2} T}{2 m}} c_{n} e^{i n \theta}$. Show that the dynamics localizes. More precisely, if $R^{k}(\psi)=\sum_{n} c_{n}^{k} e^{i n \theta}$ then for some $C=C(\psi)$ we have $\sum_{|n| \leq C}\left|c_{n}^{k}\right|^{2}>1 / 2$ for all $k$. It is necessary to avoid resonances $[\mathbf{C C I F}]$. Computer experiments support this statement $[\mathbf{G r}]$. See [F2], [We] for rigorous results for a simplified model.

Question 2.13. Define chaos in the setting of infinite dimensional complex dynamics. See [F2].

Question 2.14. Suppose that $f$ is an endomorphism of $\mathbb{P}^{k}$ or an automorphism of $\mathbb{C}^{k}$ with an attracting basin $\Omega$ for an attracting fixed point $p$, $f(p)=p$. Let $\delta>0$. For $q \in \Omega$, $\operatorname{dist}(q, \partial \Omega)>\delta, \operatorname{dist}(q, p)>\delta$, i.e. $q \in \Omega_{\delta}^{*}$. Find $N(\delta)$ so that no orbit $\left\{q, f(q), \ldots, f^{N}(q)\right\} \subset \Omega_{\delta}^{*}$. Example $\left(z^{2}+\epsilon w, z\right)$ with $p=0$. The question here is to get explicit estimates of $N(\delta)$ in terms of $f, p$ and $\delta$. The question is to have concrete estimates on how many iterates you need to get close to the fixed point if you start near the boundary of the basin.

\subsubsection{Julia sets, currents.}

Question 2.15. Let $f$ be a holomorphic selfmap of $\mathbb{P}^{k}$. Let $\Omega(f)$ be the nonwandering set. Recall that a point is nonwandering if for every neighborhood $U$ of $p$, there is an $n>1$ such that $f^{n}(U) \cap U \neq \emptyset$. The question is to describe $\Omega(f)$. 
Define a Siegel set as a closed analytic set $X$ in an open set $V \subset$ $\mathbb{P}^{k}$ such that there is a subsequence $n_{i} \rightarrow \infty$ with $f_{\mid X}^{n_{i}} \rightarrow \operatorname{Id}_{\mid X} . \quad$ Is $\Omega(f)$ the closure of the periodic points together with Siegel analytic sets? See $[\mathbf{F S 3}],[\mathbf{F S 6}],[\mathbf{B S 2}]$. Can there be a counterexample for entire maps on $\mathbb{C}^{2}$ ? (Probably yes.)

Question 2.16. Let $f$ be a holomorphic selfmap of a complex manifold $M$. Is the closure of the repelling periodic orbits open in $\Omega(f)$ ? Can there be a counterexample for entire maps in $\mathbb{C}^{n}$ ? Could there be a sequence of oscillating orbits converging to a repelling point? Can a sequence of saddles converge to a repelling point for a holomorphic selfmap on $\mathbb{C}^{2}$ ? (This cannot happen for biholomorphisms because for the inverse map the repelling point becomes attracting.) We say that the orbit of a point $p$ is oscillating if some subsequence is uniformly bounded and some other subsequence converges to infinity. For construction of oscillating domains see [FS5]. On dynamics of transcendental maps see [FS7], [FS9].

Question 2.17. Let $f$ be a polynomial map on $\mathbb{C}^{k}$ of topological degree $d_{t}$, which extends to a holomorphic map on $\mathbb{P}^{k}$. Let $\omega$ be the Kähler form on $\mathbb{P}^{k}$. It is known that

$$
\frac{\left(f^{n}\right)^{*} \omega^{k}}{d_{t}^{n k}} \rightarrow \mu
$$

where $\mu$ is a mixing probability measure [FS1]. The measure $\mu$ is the unique measure of maximal entropy [BD2] and repelling periodic points are dense in $S_{\mu}$, the support of $\mu$ [BD1]. So $S_{\mu} \subset \Omega(f)$. Is the support of $\mu$ open in $\Omega(f)$ ? It is the case when $S_{\mu}$ is hyperbolic.

Recall that the Hausdorff dimension of a probability measure $\nu$ is the minimal Hausdorff dimension of a Borel set with $\nu(B)=1$.

For a polynomial map in $\mathbb{C}, \mu$ coincides with the harmonic measure with respect to $\infty$ of the compact $K_{P}:=\left\{z ; P^{n}(z)\right.$ is bounded $\}$. It follows from the work of Makarov [Ma], Bishop-Jones $[\mathbf{B J}]$, Wolff $[\mathbf{W}]$ that the Hausdorff dimension of $\mu$ is 1 . How does this result extend to polynomial maps on $\mathbb{C}^{k}$ that are holomorphic on $\mathbb{P}^{k}$ ?

What is the Hausdorff dimension of $\mu$ for an endomorphism of $\mathbb{P}^{k}$ ? $A$ first case is to study small perturbations $\left(z^{2}+\epsilon w, w^{2}+\delta z\right)$. When is $\mu$ absolutely continuous with respect to Lebesgue measure.

Question 2.18. The Fatou set of an endomorphism $f$ of $\mathbb{P}^{k}$ is the maximal open set where the sequence $f^{n}$ is locally equicontinuous. The Julia set is the complement. It coincides with the support of the positive 
closed current $T=\lim \frac{\left(f^{n}\right) * \omega}{d^{n}}$, here $d$ is the algebraic degree of $f[\mathbf{F S 1}$, [FS4]. The Julia set $\mathcal{J}$ of an endomorphism on $\mathbb{P}^{k}$ is not in general contained in the nonwandering set (contrary to what happens when $k=1$ ). Let $\mathcal{J}_{0}:=\Omega\left(f_{J}\right)$. What can be said about the Hausdorff dimension of $\mathcal{J}, \mathcal{J}_{0}, \mathcal{J} \backslash \mathcal{J}_{0}$ ? Describe $\mathcal{J}_{0} \backslash \operatorname{Supp}(\mu)$. When $k=1, J \subset J_{0}$.

Question 2.19. Given an endomorphism of $\mathbb{P}^{k}$, when is the current $T$ extremal? This is true for maps of the form $\left[P_{1}: \cdots: P_{k}: t^{d}\right][\mathbf{B e S i}]$. Here the polynomials $P_{j}$ are homogeneous polynomials of degree $d$ in $z$ and do not contain a term in $t$. Jonsson has observed that for Ueda type examples the Green current is not extremal, for example, this is the case for the map $\left[z^{2}: w^{2}-2 z t: t^{2}\right]$.

Question 2.20. We say that an endomorphism of $\mathbb{P}^{k}$ is critically finite if each irreducible component of the critical hypersurface is a preperiodic set. Find families of endomorphisms of $\mathbb{P}^{2}$ of critically finite maps [FS12], [J].

Question 2.21. For $f$ an endomorphism of $\mathbb{P}^{k}$, when is $\operatorname{Supp}(\mu)=\mathbb{P}^{k}$ ? For how many maps? Say, find the Hausdorff dimension of this set of maps. When is $\mu$ absolutely continuous with respect to Lebesgue measure. When $k=1, S_{\mu}=\mathbb{P}^{1}$ if and only if the Julia set of $f$ is $\mathbb{P}^{1}$. If one considers rational maps of degree $d \geq 2$ on $\mathbb{P}^{1}$, this happens for a set of positive Lebesgue measure $[\mathbf{R}]$.

Question 2.22. Let $f$ be a holomorphic endomorphism of $\mathbb{P}^{k}, k>1$. $A$ closed set $A$ is attracting if there is an open set $U(A)$ such that $f(U(A)) \subset \subset U(A), \cap f^{n}(U)=A$. $A$ is an attractor if it is attracting and has a dense orbit. (Sometimes one assumes instead that it is chain transitive, i.e. $\delta$-pseudo-orbits are dense.) The attractor is non-trivial if it is not a periodic orbit or the whole space.

Show that non trivial attractors are robust in $\mathbb{P}^{2}$, i.e. for the endomorphisms $\left(f_{c}\right)$ on $\mathbb{P}^{2}$, find a set of positive measure in the c-space with a nontrivial attractor.

Give estimates for the Hausdorff dimension of A. For examples of non trivial attractors on $\mathbb{P}^{2}$ see $[\mathbf{J W}],[\mathbf{F S} 8]$. There are some estimates on Hausdorff dimension of attractors in [FS8].

Question 2.23. Does there exist a Hénon map $f=(g, h), f^{n}=\left(g_{n}, h_{n}\right)$ with the following property: There is a $p=(x, y) \in S_{\mu}$ such that for all $0<\epsilon<<1$ and every $q \in S_{\mu}$, there is an integer $n$ so that $\| g_{n}(p)-$ $g_{n}(q) \|<\epsilon$ ? This question arises naturally in the study of attractors: A collision-attractor absorbs all points whose orbits are closer than some radius for some iterate. If one considers $x$ as a space variable, and $y$ 
as a momentum variable, one measures distance using the first variable only. If one replaces the inequality by $\left\|f^{n}(p)-f^{n}(q)\right\|<\epsilon$, there is no such $p,($ see $[\mathbf{B F}])$.

Question 2.24. Let $f(z, w)=\left(e^{i \theta} z, e^{-i \theta} w\right)+$ higher order terms be a generalized Hénon map. Give diophantine conditions on $\theta$ for which $f$ has two Siegel discs, $D_{1}, D_{2} \subset J^{+} \cap J^{-}$through the origin and tangent to the axes. Recall that generalized Hénon maps are finite compositions of Hénon maps. They are the dynamically interesting polynomial biholomorphisms of $\mathbb{C}^{2}$.

Question 2.25. Consider $\mathcal{S}_{\mathbb{R}}$, the class of holomorphic symplectomorphisms of $\mathbb{C}^{2 k}$ preserving $\mathbb{R}^{2 k}$. $f: \mathbb{C}^{2 k} \rightarrow \mathbb{C}^{2 k}, f^{*} \omega=\omega$, where $\omega=$ $\sum_{j=1}^{k} d z_{j} \wedge d w_{j}$ and $f\left(\mathbb{R}^{2 k}\right)=\mathbb{R}^{2 k}, z_{j}=x_{j}+i x_{j}^{\prime}, w_{j}=y_{j}+i y_{j}^{\prime}$ with Whitney fine topology, $\mathcal{S}_{\mathbb{R}}$ is a Baire space. For $f \in \mathcal{S}_{\mathbb{R}}$, let $K_{f}^{\mathbb{R}}:=\left\{(x, y) \in \mathbb{R}^{2 k} ;\left\{f^{n}(x, y)\right\}_{n}\right.$ is bounded $\}$. Prove that the set $\mathcal{S}_{\mathbb{R}}^{\prime}$ of $f \in \mathcal{S}_{\mathbb{R}}$ such that $K_{f}^{\mathbb{R}}$ is of empty interior in $\mathbb{R}^{2 k}$ is a $G_{\delta}$ dense set $[\mathbf{F S 7}]$, [FS9].

Question 2.26. Let $f(z, w)=\left(e^{i \theta} z, e^{i \psi} w\right)+$ higher order terms. Study the dynamics near the origin. Hakim-Abate-Weickert have studied the case where $f$ is tangent to the identity $[\mathbf{H a 1}],[\mathbf{H a 2}][\mathbf{A b}],[\mathbf{W e}]$.

Question 2.27 (D. Burns). Suppose that $f: X_{\mathbb{C}} \rightarrow X_{\mathbb{C}}$ is an endomorphism on a projective variety over $\mathbb{C}$. Assume that $f$ has "large" $d y$ namics. For example $f$ has positive entropy or a "large" non wandering set. Consider now $X_{(k)}$ as a variety over a number field $k$ such that $[k: \mathbb{Q}]<\infty$. Does largeness of the dynamics imply largeness of the set of rational points in $X_{(k)}$ ? For example, are rational points Zariski dense in $X_{\mathbb{C}}$ ? (J. B. Bost). The existence of mappings with large dynamics should imply arithmetic properties of $X_{(k)}$.

Question 2.28. Let $K$ be a hyperbolic solenoid with stable dimension 2 . How to get a stable current? Are the stable leaves biholomorphic to $\mathbb{C}^{2}$ ?

For endomorphisms on $\mathbb{P}^{k}$ see $[\mathbf{F S 4}],[\mathbf{F S 2}],[\mathbf{F S 8}],[\mathbf{F S 1 1}],[\mathbf{F 1}],[\mathbf{S 1}]$, [BD1], [BD2], [Ga], [DS].

\subsection{Continuous dynamics.}

Question 2.29. Is there a compact set $K \subset \mathbb{P}^{2}$ which is laminated by smooth holomorphic curves, except a compact curve? There are such compacts in $\mathbb{P}^{3}$. See survey by Ghys $[\mathbf{G h}]$. There is no closed $(1,1)$ current directed by the lamination in $\mathbb{P}^{2}$. (I.e. if locally the current is of the form $\int d \mu_{\theta}\left[V_{\theta}\right]$, then the lamination is a compact curve.) See $[\mathbf{H M}]$. 
The origin of the question seems to be related to a Poincaré-Bendixson Theorem for holomorphic foliations on $\mathbb{P}^{2},[\mathbf{C L S}]$. More precisely, let $\mathcal{F}$ be a holomorphic foliation on $\mathbb{P}^{2}$. The singularity set of $\mathcal{F}, \operatorname{Sing} \mathcal{F}$ is never empty. The question is whether the closure of any leaf $L$ intersects Sing $\mathcal{F}$. If not $\bar{L}$ will be laminated by smooth holomorphic curves $[\mathbf{C L S}]$.

Question 2.30. Let $P_{N}$ consist of the holomorphic polynomials of degree at most $N$ in $\mathbb{C}^{2 k}, k \geq 2, N \geq 2$. Show that for almost every $P_{N}$ and almost every point $z \in \mathbb{C}^{2 k}$ the orbit of the Hamiltonian vector field $X_{P_{N}}$ is unbounded. Recall that when $h$ is an entire map in $\mathbb{C}^{2 k}$,

$$
X_{h}:=\left(-\frac{\partial h}{\partial w_{1}}, \ldots,-\frac{\partial h}{\partial w_{k}}, \frac{\partial h}{\partial z_{1}}, \ldots, \frac{\partial h}{\partial z_{k}}\right) .
$$

[FS7], [FS9] contain results on Hamiltonian vector fields and dynamical symplectomorphisms. (When $k=1$, see $[\mathbf{D u}]$.)

\section{Several complex variable problem list}

We divide the questions into three sets, depending whether they fit most naturally together with $\bar{\partial}$, the Levi Problem or Holomorphic Mappings

\section{1. $\bar{\partial}$.}

Question 3.1 (Henkin). Let $X$ be a normal analytic set of pure dimension $p$ in the unit ball $B \subset \mathbb{C}^{n}$. Let $f \in \mathcal{C}_{(0,1)}^{\infty}(B)$. Assume $\bar{\partial}\left(\operatorname{Id}_{\mid \operatorname{Reg}(X)}^{*} f\right)=$ 0 . Does there exist $u \in \mathcal{C}_{(0,0)}^{\infty}(B)$ such that $\bar{\partial}\left(\operatorname{Id}_{\operatorname{Reg}(X)}^{*} u\right)=\left(\operatorname{Id}_{\mid \operatorname{Reg}(X)}^{*} f\right)$ ? Here $\operatorname{Id}_{\operatorname{Reg}(X)}$ is the inclusion map from $\operatorname{Reg}(X)$ to $\mathbb{C}^{n}$. See $[\mathbf{H e P}],[\mathbf{M}]$, [AG1], [AG2].

Question 3.2. Let $\Omega$ be a weakly pseudoconvex domain with smooth boundary in $\mathbb{P}^{n}, n \geq 2$. Let $f \in \mathcal{C}_{(0,1)}^{\infty}(\bar{\Omega})$ be a $\bar{\partial}$-closed $(0,1)$ form. Does there exist a smooth solution $u \in \mathcal{C}^{\infty}(\bar{\Omega})$ to the equation $\bar{\partial} u=f$ ?

Question 3.3 (Lempert-Henkin). Let $D_{N}=\left\{z \in \mathbb{C}^{N} ; \sum_{j=1}^{N}\left|z_{j}\right|<1\right\}$. Prove (or find a counterexample) that for $f \in \mathcal{C}_{(0,1)}\left(\bar{D}_{N}\right)$ with $\bar{\partial} f=0$ there is $u \in \mathcal{C}\left(\bar{D}_{N}\right)$ such that $\bar{\partial} u=f$ and

$$
\|u\|_{\infty} \leq C\|f\|_{\infty} .
$$

The key point here is that $C$ should be independent of $N$. One can ask the same question for $D_{N}^{2}=\left\{z \in \mathbb{C}^{N} ; \sum_{j=1}^{N}\left|z_{j}\right|^{2}<1\right\}[\mathbf{L}]$. 
Question 3.4 (Berndtsson). Let $\phi$ be a bounded strictly plurisubharmonic function in the unit ball $\mathbb{B} \subset \mathbb{C}^{n}$. Let $\Omega=i \partial \bar{\partial} \phi$, the Kähler form associated to $\phi$. Let $f$ be a $(0,1)$ form on $B$ such that $|f|_{\Omega}^{2}+|\partial f|_{\Omega} \leq C$. The subscript means that the norm for $f$ and $\partial f$ are measured with respect to $\Omega$. Does the equation $\bar{\partial} u=f$ have a bounded solution in B? The question is related to the Corona theorem in several variables $[\mathbf{B e}]$.

Question 3.5 (Edited by Sophia Vassiliadou). Let $X$ be a closed analytic set of pure dimension $p$ in $\mathbb{C}^{n+p}$ with singularities. Let $\operatorname{Reg}(X)=$ $X \backslash \operatorname{Sing}(X)$ denote the set of smooth points of $X$. Give $\operatorname{Reg}(X)$ the metric induced by the imbedding $\operatorname{Reg}(X) \hookrightarrow \mathbb{C}^{n+p}$.

(a) Let $f \in L_{(0, q)}^{2}(\operatorname{Reg}(X)), 1 \leq q \leq p, \bar{\partial} f=0$ in the weak sense in $L_{0, q+1}^{2}(\operatorname{Reg}(X))$. Find the obstructions to solving $\bar{\partial} u=f$ in the weak sense in $L_{0, q}^{2}(\operatorname{Reg}(X))$. Recall that $\bar{\partial} u=f$ in the weak sense in $L_{0, q}^{2}(\operatorname{Reg}(X))$ if and only if $u \in L_{0, q-1}^{2}(\operatorname{Reg}(X))$ and for all $\psi \in \mathcal{C}_{p, p-q}^{\infty}(\operatorname{Reg}(X))$, compactly supported in $\operatorname{Reg}(X)$ we have

$$
\int_{\operatorname{Reg}(X)} u \wedge \bar{\partial} \psi=(-1)^{q} \int_{\operatorname{Reg}(X)} f \wedge \psi .
$$

(b) Let $h \in L_{(\alpha, \beta)}^{2}(\operatorname{Reg}(X))$. We say that $h$ belongs to the domain of $\bar{\partial}$ with Dirichlet boundary conditions $-h \in \operatorname{Dom} \bar{\partial}_{D}$ - if and only if there exist $h_{n} \in \mathcal{C}_{\alpha, \beta}^{\infty}(\operatorname{Reg}(X))$, compactly supported in $\operatorname{Reg}(X)$ and $g \in L_{\alpha, \beta+1}^{2}(\operatorname{Reg}(X))$ such that $h_{n} \rightarrow h$ in $L_{\alpha, \beta}^{2}(\operatorname{Reg}(X))$ and $\bar{\partial} h_{n} \rightarrow g$ in $L_{\alpha, \beta+1}^{2}(\operatorname{Reg}(X))$. In that case we write $\bar{\partial}_{D} h=: g$. Let $f \in L_{(p, q)}^{2}(\operatorname{Reg}(X)), 1 \leq q \leq p$ such that $\bar{\partial}_{D} f=0$. Find the obstructions to solving $\bar{\partial}_{D} u=f$, (i.e. obstructions to obtaining $u_{n} \in \mathcal{C}_{(p, q-1)}^{\infty}(\operatorname{Reg}(X))$, compactly supported in $\operatorname{Reg}(X)$ such that $u_{n} \rightarrow u$ in $L_{(p, q-1)}^{2}(\operatorname{Reg}(X))$ and $\bar{\partial} u_{n} \rightarrow f$ in $\left.L_{(p, q)}^{2}(\operatorname{Reg}(X))\right)$.

In $[\mathbf{P S}]$, (a) and (b) are solved when $X$ is a projective surface with isolated singularity. They also computed obstructions to solving $\bar{\partial}$ weakly for $\bar{\partial}$ closed $(p, q)$ forms when $X$ is a projective variety of dimension $p$, as well as obstructions to solving $\bar{\partial}_{D}$ for $(0, q), \bar{\partial}_{D}$-closed forms again when $X$ is a projective variety of dimension $p$.

See [BeSi] for an alternative point of view: Solving $\bar{\partial}$ on positive currents (especially positive, closed currents of bidegree $(1,1)$ ).

See [DFV] for obstructions to solving $\bar{\partial}$ weakly for $(0,1)$ forms near 2 -dimensional isolated singularities in $\mathbb{C}^{n}, n \geq 3$. 
Question 3.6. (a) Let $T$ be a positive closed current of bidimension $(p, p)$ in $\mathbb{C}^{n}, 1 \leq p<n-1$. Is $T$ a weak limit of $c_{j}\left[V_{j}\right], \operatorname{dim} V_{j}=p$, analytic and $c_{j}>0$.

(b) Can $\overline{V_{j}} \rightarrow$ Supp $T$ in the Hausdorff metric? The case when $T$ is of bidimension $(n-1, n-1)$ (i.e. $\left.\operatorname{dim} V_{j}=n-1\right)$ is classical. For the recent refinements see Demailly [D1], [D2], Duval-Sibony [DS1], Guedj $[\mathbf{G u}]$ for currents in complex manifolds.

\subsection{Levi problem.}

Question 3.7. Let $X \subset \mathbb{C}^{n}, 0 \in X$ be a complex analytic set with an isolated normal singularity, $K$ a compact subset of $X \backslash(0), 0 \in \hat{K}$, the polynomially convex hull of $K$. Suppose that $L$ is compact in $X$, containing $K$ in its relative interior. Does $\hat{L}$ contain a neighborhood of 0 in $X$ ? This is equivalent to a Runge problem: If $\left(\Omega_{t}\right)_{t \in(-1,1)}$ is a continuously increasing family of Stein open subsets of $X, 0 \in X \backslash \Omega_{t}$, $t \leq 0,0 \in \Omega_{t}, t>0$. Is $\Omega_{t_{1}}$ Runge in $\Omega_{t_{2}}$ if $t_{1} \leq 0<t_{2}$ ? See $[\mathbf{F N}]$.

Question 3.8. Suppose that $X$ is a singular Stein space, $\Omega \subset \subset X$. Suppose that for all $p \in \partial \Omega$ there is an open neighborhood $U(p)$ so that $U \cap \Omega$ is Stein. Is $\Omega$ Stein? [FN], [Si1].

Question 3.9. Let $\Omega \subset \subset M$ be a compact complex manifold, $\partial \Omega$ connected. Suppose that $f$ is holomorphic in a neighborhood of $\partial \Omega$. Under which natural conditions does $f$ extend to $\Omega$ or its complement? Exclude $M=\Delta * N, N$ compact. It is true if $\Omega$ is Stein. Same question when $f$ is $C R$ on $\partial \Omega$. When $M$ is $\mathbb{P}^{k}$, Sarkis $[\mathbf{S a}]$ has shown that $\mathcal{C}^{1} C R$-functions on $\partial \Omega$ extend to one side.

Question 3.10. Does there exist a $\mathcal{C}^{2}$ Levi flat hypersurface in $\mathbb{P}^{2}$ ? Same question for topological hypersurfaces, i.e. locally graphs which are locally union of disjoint holomorphic discs. Describe all $\mathcal{C}^{\infty}$ Levi flat hypersurfaces in a complex torus. This is related to Question 2.6. See Siu $[\mathbf{S i 2}]$ for the $\mathcal{C}^{\infty}$ case.

Question 3.11 (Henkin). (a) Construct a strictly pseudoconvex domain $M$ with smooth boundary in a complex 2-dimensional surface in $\mathbb{P}^{3}$ which intersects every compact Riemann surface in $\mathbb{P}^{3}$. B. Fabre [Fab1, p. 88] has constructed a real 3-dimensional $M \subset$ $\mathbb{P}^{3}$ with Levi-flat boundary and which intersects any compact Riemann surface.

(b) Let $V$ be an algebraic hypersurface in $\mathbb{P}^{3}$. Let $M$ be a strictly pseudoconvex domain in $V$. Is there a Riemann surface $S$ in $\mathbb{P}^{3}$ with $S \cap V \subset V \backslash M$ ? 
Question 3.12 (Gromov). Let $\mathcal{A}_{n-1}$ be the space of smooth analytic varieties of pure dimension $n-1$ in $\mathbb{P}^{n}$. When $n=2$, F. A. Bogomolov [Bo] has constructed a sequence of Riemann surfaces $\left(V_{j}\right)$ in $\mathcal{A}_{1}$ such that $\sup _{j} \operatorname{diam}\left(V_{j}\right)=\infty$. Here the diameter in $V_{j}$ is computed with respect to the Hermitian metric induced from $\mathbb{P}^{n}$. Does a similar phenomenon occur for subvarieties in $\mathcal{A}_{n-1}, n>2$ ?

Question 3.13. Let $M$ be a totally real compact manifold in $\mathbb{C}^{n}$, $\operatorname{dim}(M)_{\mathbb{R}}=n$. Does it bound a smooth Riemann surface, smooth up to the boundary? For $\epsilon>0$, there is a Riemann surface with boundary in $M(\epsilon)[\mathbf{V i}]$. Here $M(\epsilon)=\{z ; \operatorname{dist}(z, w)<\epsilon\}$. Gromov showed the existence of discs when $M$ is Lagrangian, for certain Kähler forms. There are examples without discs. Gromov $[\mathbf{G}]$, Alexander $[\mathbf{A l}]$, DuvalSibony [DS1], [DS2], Viterbo [Vi].

Question 3.14. Let $M$ be a smooth projective manifold, $\Omega$ a proper open subset, locally Stein at the boundary. When is $\Omega$ holomorphically convex? See $[\mathbf{N}]$. (Grauert found a counterexample which is a Levi flat domain on a complex torus, $\{(z, w) ; a<\Re, z<b\}$ with irrational slope. In Grauert's domain all holomorphic functions are constant.) So we assume that $\partial \Omega$ has 1 strongly pseudoconvex boundary point. See [Si1].

Question 3.15 (L. Nirenberg). Let $D \subset \mathbb{C}^{n}, n \geq 2$, be a domain with smooth boundary. Let $\gamma$ be a smooth curve in $\partial D$ transverse to the complex tangent space $T_{p}^{\mathbb{C}}(\partial D)$ for every $p$ in $\gamma$. Prove that if $f \in \mathcal{C}^{\infty}(\bar{D})$, holomorphic on $\Omega$ and all derivatives (in all directions) $f^{(k)}$ vanish on $\gamma \forall k \in \mathbb{Z}_{+}^{n}$, then $f \equiv 0$. When $\gamma$ is real analytic, the result and some consequences are in $[\mathbf{N i}]$.

\subsection{Holomorphic mappings.}

Question 3.16. Can one embed all Stein Riemann surfaces as closed complex submanifolds of $\mathbb{C}^{2}$ ? (See $[\mathbf{G S}]$.)

Question 3.17. Let $\Omega$ be a smoothly bounded pseudoconvex domain with noncompact automorphism group $G$. Does $G$ contain $\mathbb{R}$ ?

When $\Omega$ is convex, see $[\mathbf{F r}]$.

Question 3.18 (Pinchuk). Let $\Sigma$ be a real analytic hypersurface, $\partial \Omega$ a real analytic strictly pseudoconvex, nonspherical hypersurface or simply connected. Suppose $f$ is a holomorphic map defined on $\Sigma$ in a neighborhood of a strictly pseudoconvex point, with values in $\partial \Omega$. Can $f$ be extended along any curve?

Some special cases are considered in $[\mathbf{P i}],[\mathbf{S h}],[\mathbf{B P}]$. 
Question 3.19. Let $\Sigma$ be a real analytic hypersurface of finite type. Let $X=\sum a_{j} \frac{\partial}{\partial z_{j}},\left(a_{j}\right.$ holomorphic) be a holomorphic vector field, $\Re X$ tangent to $\Sigma$. Classify these $X$ and use them to classify $\Sigma$. The case of convex domains with compact automorphism groups is studied in $[\mathbf{B P}]$.

Question 3.20. Let $f: \Omega \rightarrow V$ be biholomorphic, $\Omega, V$ bounded open sets in $\mathbb{C}^{n}$ with $\mathcal{C}^{\omega}$ boundary. Does $f$ extend continuously to the boundary? How about $\mathcal{C}^{\infty}$ domains? Do there exist pseudoconvex $\Omega, V$, biholomorphism, not extending smoothly to the boundary? Local geometry to analyze: $\left\{\left(\Re z_{0}+\left|z_{1}\right|^{2}-\left|z_{2}\right|^{2}\right)^{2}+\Re z_{4}<0\right\}$. (All known methods fail.)

Question 3.21. Jacobian Conjecture: Let $f: \mathbb{C}^{2} \rightarrow \mathbb{C}^{2}$ be a polynomial mapping. Assume that the Jacobian of $f$ does not vanish. Then $f$ is invertible $[\mathbf{B C W}],[\mathbf{V}],[\mathbf{O}]$.

Question 3.22. Let $\Omega$ be a domain with real analytic boundary, $p \in \partial \Omega$, $p$ not in the envelope of holomorphy, $p$ of finite type. Does there exist an $\epsilon>0$ so that the infinitesimal Kobayashi metric satisfies the estimate

$$
F(q, X) \geq \frac{|X|}{|p-q|^{\epsilon}} ?
$$

This will be useful to prove that proper, holomorphic maps $\Omega \rightarrow \Omega^{\prime}$ extend contiuously to $\bar{\Omega}$.

Question 3.23 (Kobayashi hyperbolicity problem). Let $X \subset \mathbb{P}^{n}$ be $a$ generic hypersurface of degree $\geq 2 n+1$. Is $\mathbb{P}^{n} \backslash X$ Kobayashi hyperbolic? More precisely, is any holomorphic map $\mathbb{C} \rightarrow \mathbb{P}^{n} \backslash X$ constant? A lot of work has been done in this direction. We only mention Siu-Yeung $[\mathbf{S Y}]$, Demailly-El Goul [DG] and Noguchi, Winkelman, Yamanoi [NWY].

Question 3.24 (Diederich/Pinchuk). Let $H \subset B^{n}, H$ a $\mathcal{C}^{\infty}$ hypersurface of finite type. Let $X_{n}$ be a sequence of closed subvarieties of $B^{n}$, $0 \in X_{n} \cap H$. Can the cluster set of $X_{n}$ be a contained in $H$ ? When $H$ is pseudoconvex, then every point has a plurisubharmonic barrier [S2] and hence the cluster set is not contained in $\mathrm{H}$.

\section{References}

[Ab] M. AвAte, The residual index and the dynamics of holomorphic maps tangent to the identity, Duke Math. J. 107(1) (2001), 173-207.

[A] L. V. Ahlfors, "Conformal invariants: topics in geometric function theory", Mc Graw-Hill Book Co., New York, 1973.

[Al] H. Alexander, Gromov's method and Bennequin's problem, Invent. Math. 125(1) (1996), 135-148. 
[AG1] V. Ancona and B. Gaveau, Differential forms and resolutions on certain analytic spaces. I. Irreducible excepcional divisor, Bull. Sci Math. 116(3) (1992), 307-324.

[AG2] V. Ancona and B. Gaveau, Differential forms and resolutions on certain analytic spaces. II. Flat resolutions, Canad. J. Math. 44(4) (1992), 728-749.

[BCW] H. BAss, E. H. Connell And D. Wright, The Jacobian conjecture: reduction of degree and formal expansion of the inverse, Bull. Amer. Math. Soc. (N.S.) 7(2) (1982), 287-330.

[BLS] E. Bedford, M. Lyubich and J. Smillie, Polynomial diffeomorphisms of $\mathbb{C}^{2}$. IV. The measure of maximal entropy and laminar currents, Invent. Math. 112(1) (1993), 77-125.

[BP] E. Bedford and S. I. Pinchuk, Convex domains with noncompact groups of automorphisms, (Russian), Mat. Sb. 185(5) (1994), 3-26.

[BS1] E. Bedford and J. Smillie, Polynomial diffeomorphisms of $\mathbb{C}^{2}$ : currents, equilibrium measure and hyperbolicity, Invent. Math. 103(1) (1991), 69-99.

[BS2] E. Bedford AND J. Smillie, Polynomial diffeomorphisms of $\mathbb{C}^{2}$. II. Stable manifolds and recurrence, J. Amer. Math. Soc. 4(4) (1991), 657-679.

[BT] E. Bedford and B. A. Taylor, The Dirichlet problem for a complex Monge-Ampére equation, Invent. Math. 37(1) (1976), $1-44$.

[Be] B. Berndtsson, Uniform estimates with weights for the $\bar{\partial}$ equation, J. Geom. Anal. 7(2) (1997), 195-215.

[BeSi] B. Berndtsson and N. Sibony, The $\bar{\partial}$ equation on a positive current, Preprint (2000).

[BJ] C. J. Bishop and P. W. Jones, Harmonic measure and arclength, Ann. of Math. (2) 132(3) (1990), 511-547.

[Bo] F. A. Bogomolov, On the diameter of plane algebraic curves, Math. Res. Lett. 1(1) (1994), 95-98.

[BF] A. M. Bonifant and J. E. Forness, Attractors, Preprint (2001).

[BD1] J.-Y. Briend and J. Duval, Exposants de Lyapunoff et distribution des points périodiques d'un endomorphism de $\mathbb{P}^{k}$, Acta Math. 182(2) (1999), 143-157.

[BD2] J.-Y. BRIEnd AND J. Duval, Deux caractérisations de la mesure d'equilibre d'un endomorphisme de $\mathbb{P}^{k}$, Preprint (2000). 
[Bu] G. T. Buzzard, Infinitely many periodic atractors for holomorphic maps of 2 variables, Ann. of Mat. (2) 145(2) (1997), 389-417.

[CLS] C. Camacho, A. Lins Neto and P. Sad, Minimal sets of foliations on complex projective spaces, Inst. Hautes Études Sci. Publ. Math. 68 (1988), 187-203.

[CG] L. Carleson and T. W. Gamelin, "Complex Dynamics", Universitext: Tracts in Mathematics, Springer-Verlag, New York, 1993.

[CCIF] G. Casati, B. V. Chirikov, F. M. Izrăllev and J. Ford, Stochastic behavior of a quantum pendulum under a periodic perturbation, in: "Stochastic behaviour in classical and quantum Hamiltonian systems" (Volta Memorial Conf., Como, 1977), Lecture Notes in Physics 93, Springer, Berlin-New York, 1979, pp. 334-352.

[D1] J.-P. Demailly, Courants positifs extrêmaux et conjecture de Hodge, Invent. Math. 69(3) (1982), 347-374.

[D2] J.-P. Demailly, Pseudoconvex-concave duality and regularization of currents, in: "Several complex variables" (Berkeley, CA, 1995-1996), Math. Sci. Res. Inst. Publ. 37, Cambridge Univ. Pres, Cambridge, 1999, pp. 233-271.

[DG] J.-P. Demailly and J. El Goul, Hyperbolicity of generic surfaces of high degree in projective 3-space, Amer. J. Math. 122(3) (2000), 515-546.

[DFV] K. Diederich, J. E. Forness and S. K. Vassiliadou, in preparation.

[DS] T.-C. Dinh And N. Sibony, Dynamique d'endomorphismes holomorphes, in preparation.

[Du] R. Dujardin, Dynamics of polynomial Hamiltonian vector fields in $\mathbb{C}^{2 k}$, Preprint.

[DS1] J. Duval and N. Sibony, Polynomial convexity, rational convexity, and currents, Duke Math. J. 79(2) (1995), 487-513.

[DS2] J. Duval and N. Sibony, Hulls and positive closed currents, Duke Math. J. 95(3) (1998), 621-633.

[Fab1] B. FABRE, Nouvelles variations autour d'un théorème d'Abel, Lie, Thesis, Paris 6 (2000).

[Fab2] B. FabRe, Sur l'intersection d'une surface de Riemann avec des hypersurfaces algébriques, C. R. Acad. Sci. Paris Sér. I Math. 322(4) (1996), 371-376. 
[F1] J. E. FORNÆSS, "Dynamics in several complex variables", CBMS Regional Conference Series in Mathematics 87, Published for the Conference Board of the Mathematical Sciences, Washington, DC; by the American Mathematical Society, Providence, RI, 1996.

[F2] J. E. Fornæss, Infinite dimensional complex dynamics: quasiconjugacies, localization and quantum chaos, Discrete Contin. Dynam. Systems 6(1) (2000), 51-60.

[FN] J. E. Forness and R. NARASImHan, The Levi problem on complex spaces with singularities, Math. Ann. 248(1) (1980), $47-72$.

[FS1] J. E. Forness and N. Sibony, Complex dynamics in higher dimension. II, in: "Modern methods in complex analysis" (Princeton, NJ, 1992), Ann. of Math. Stud. 137, Princeton Univ. Press, Princeton, NJ, 1995, pp. 135-182.

[FS2] J. E. Forness And N. Sibony, Hyperbolic maps on $\mathbb{P}^{2}$, Math. Ann. 311(2) (1998), 305-333.

[FS3] J. E. FornæsS AND N. Sibony, Complex Hénon mappings in $\mathbb{C}^{2}$ and Fatou-Bieberbach domains, Duke Math. J. 65(2) (1992), $345-380$.

[FS4] J. E. Forness And N. SibOnY, Oka's inequality for currents and applications, Math. Ann. 301(3) (1995), 399-419.

[FS5] J. E. Forness And N. Sibony, Fatou and Julia sets for entire mappings in $\mathbb{C}^{k}$, Math. Ann. 311(1) (1998), 27-40.

[FS6] J. E. FornæsS AND N. SibOny, Classification of recurrent domains for some holomorphic maps, Math. Ann. 301(4) (1995), $813-820$.

[FS7] J. E. FornæsS AND N. Sibony, Holomorphic symplectomorphisms in $\mathbb{C}^{2 p}$, Duke Math. J. 82(2) (1996), 309-317.

[FS8] J. E. Fornæss AND N. Sibony, Dynamics in $\mathbb{P}^{2}$ (examples), in: "Laminations and foliations in dynamics, geometry and topology" (Stony Brook, NY, 1998), Contemp. Math. 269, Amer. Math. Soc., Providence, RI, 2001, pp. 47-85.

[FS9] J. E. Fornæss and N. Sibony, Complex dynamics in higher dimension, in: "Several complex variables" (Berkeley, CA, 1995-1996), Math. Sci. Res. Inst. Publ. 37, Cambridge Univ. Press, Cambridge, 1999, pp. 273-296.

[FS10] J. E. Fornæss And N. Sibony, Complex dynamics in higher dimensions, in: "Complex potential theory" (Montreal, PQ, 1993), NATO Adv. Sci. Inst. Ser. C Math. Phys. Sci. 439, Kluwer Acad. Publ., Dordrecht, 1994, pp. 131-186. 
[FS11] J. E. Fornæss And N. Sibony, Increasing sequences of complex manifolds, Math. Ann. 255(3) (1981), 351-360.

[FS12] J. E. FornæsS AND N. Sibony, Critically finite rational maps on $\mathbb{P}^{2}$, in: "The Madison Symposium on Complex Analysis" (Madison, WI, 1991), Contemp. Math. 137, Amer. Math. Soc., Providence, RI, 1992, pp. 245-260.

[FW] J. E. Forness And B. WeICKERT, Attractors in $\mathbb{P}^{2}$, in: "Several complex variables" (Berkeley, CA, 1995-1996), Math. Sci. Res. Inst. Publ. 37, Cambridge Univ. Press, Cambridge, 1999, pp. 297-307.

[Fr] S. Frankel, Complex geometry of convex domains that cover varieties, Acta Math. 163(1-2) (1989), 109-149.

[FM] S. Friedland And J. Milnor, Dynamical properties of plane polynomial automorphisms, Ergodic Theory Dynam. Systems 9(1) (1989), 67-99.

[Ga] E. A. Gavosto, Attracting basins in $\mathbb{P}^{2}$, J. Geom. Anal. 8(3) (1998), 433-440.

[Gh] E. GhYs, Laminations par surfaces de Riemann, in: "Dynamique et géométrie complexes" (Lyon, 1997), Panor. Synthèses 8, Soc. Math. France, Paris, 1999, pp. 49-95.

[GS] J. Globevnik and B. Stensønes, Holomorphic embeddings of planar domains in $\mathbb{C}^{2}$, Math. Ann. 303(4) (1995), 579-597.

[Gr] J. M. Greene, On the last KAM torus for the standard map, J. Math. Phys. 20 (1979), 1183.

[G] M. Gromov, Pseudoholomorphic curves in symplectic manifolds, Invent. Math. 82(2) (1985), 307-347.

[Gu] V. GuedJ, Approximation of currents on complex manifolds, Math. Ann. 313(3) (1999), 437-474.

[Ha1] M. Hakim, Analytic transformations of $\left(\mathbb{C}^{p}, 0\right)$ tangent to the identity, Duke Math. J. 92(2) (1998), 403-428.

[Ha2] M. HAKIM, Transformations tangent to the identity, stable pieces of manifolds, Prepublications Orsay.

[HeP] G. M. Henkin and P. L. Polyakov, The GrothendieckDolbeault lemma for complete intersections, C. R. Acad. Sci. Paris Ser. I Math. 308(13) (1989), 405-409.

[H] S. I. Hruska, Ph. D thesis, Cornell, in preparation.

[HO] J. H. Hubbard and R. W. Oberste-Vorth, Hénon mappings in the complex domain. II. Projective and inductive limits of polynomials, in: "Real and complex dynamical systems" (Hillerød, 1993), NATO Adv. Sci. Inst. Ser. C Math. Phys. Sci. 464, Kluwer Acad. Publ., Dordrecht, 1995, pp. 89-132. 
[HP] J. H. Hubbard and P. Papadopol, Superattractive fixed points in $\mathbb{C}^{n}$, Indiana Univ. Math. J. 43(1) (1994), 321-365.

[HM] S. Hurder and Y. Mitsumatsu, The intersection product of transverse invariant measures, Indiana Univ. Math. J. 40(4) (1991), 1169-1183.

[IS] F. M. IZRAĬLEv AND D. L. ŠepelJanskIĬ, Quantum resonance for the rotator in a nonlinear periodic field, (Russian), Teoret. Mat. Fiz. 43(3) (1980), 417-428.

[J] M. Jonsson, Some properties of 2-critically finite holomorphic maps of $\mathbb{P}^{2}$, Ergodic Theory Dynam. Systems 18(1) (1998), $171-187$.

[JV] M. Jonsson and D. Varolin, Preprint (2001).

[JW] M. JONSSON AND B. WeICKERT, A nonalgebraic attractor in $\mathbb{P}^{2}$, Proc. Amer. Math. Soc. 128(10) (2000), 2999-3002.

[JL] D. Jupiter And K. LiLov, Preprint (2001).

[L] L. Lempert, The Dolbeault complex in infinite dimension. II, J. Amer. Math. Soc. 12(3) (1999), 775-793.

[Ma] N. G. MAKAROV, Metric properties of harmonic measure, in: "Proceedings of the International Congress of Mathematicians", Vol. 1,2 (Berkeley, Calif., 1986), Amer. Math. Soc., Providence, RI, 1987, pp. 766-776.

[M] B. Malgrange, Systèmes differentiels à coefficients constants, Seminaire Bourbaki 15 (1962-63), no. 246.

[Mi] J. Milnor, "Dynamics in one complex variable: Introductory lectures", Friedr. Vieweg \& Sohn, Braunschweig, 1999.

[N] R. NARASimhan, The Levi problem in the theory of functions of several complex variables, in: "Proc. Internat. Congr. Mathematicians" (Stockholm (1962), Inst. Mittag-Leffler, Djursholm, 1963, pp. 385-388.

[Ni] L. Nirenberg, A certain problem of Hans Lewy, (Russian), Uspehi Mat. Nauk 29(2) (1974), 241-251.

[NWY] J. Noguchi, J. Winkelmann and K. Yamanoi, The value distribution of holomorphic curves into semi-abelian varieties, $C$. R. Acad. Sci. Paris Sér. I Math. 331(3) (2000), 235-240.

[O] S. Yu. Orevkov, An example in connection with the Jacobian conjecture, (Russian), Mat. Zametki 47(1) (1990), 127-136, 173; translation in Math. Notes 47(1-2) (1990), 82-88.

[PS] W. L. Pardon and M. A. Stern, $L^{2}-\bar{\partial}$-cohomology of complex projective varieties, J. Amer. Math. Soc. 4(3) (1991), 603-621.

[PM] R. PÉREZ-Marco, Siegel discs with smooth boundary, Preprint. 
[Pi] S. I. PINchuk, On the analytic continuation of holomorphic mappings, Mat. Sb. 27 (1975), 375-392.

[R] M. REes, Positive measure sets of ergodic rational maps, Ann. Sci. École Norm. Sup. (4) 19(3) (1986), 383-407.

$[\mathrm{Ru}] \mathrm{D}$. RuELLE, "Elements of differentiable dynamics and bifurcation theory", Academic Press, Inc., Boston, MA, 1989.

[Sa] F. SARKIS, Hartogs-Bochner type theorem in projective space, Preprint (2001).

[Sh] R. Shafikov, Analytic continuation of germs of holomorphic mappings between real hypersurfaces in $\mathbb{C}^{n}$, Michigan Math. $J$. 47(1) (2000), 133-149.

[S1] N. Sibony, Dynamique des applications rationelles de $\mathbb{P}^{k}$, in: "Dynamique et géométrie complexes" (Lyon, 1997), Panor. Synthèses 8, Soc. Math. France, Paris, 1999, pp. 97-185.

[S2] N. Sibony, Une classe de domaines pseudoconvexes, Duke Math. J. 55(2) (1987), 299-319.

[Si1] Y.-T. SiU, Pseudoconvexity and the problem of Levi, Bull. Amer. Math. Soc. 84(4) (1978), 481-512.

[Si2] Y.-T. SiU, $\bar{\partial}$ regularity for weakly pseudoconvex domains in compact Hermitian symmetric spaces with respect to invariant metrics, Preprint (2001).

[SY] Y.-T. SiU And S.-K. Yeung, Hyperbolicity of the complement of a generic smooth curve of high degree in the complex projective plane, Invent. Math. 124(1-3) (1996), 573-618.

[St] B. Stensønes, Fatou-Bieberbach domains with $\mathcal{C}^{\infty}$-smooth boundary, Ann. of Math. (2) 145(2) (1997), 365-377.

[T] M. TsusI, "Potential theory in modern function theory", Maruzen Co., Ltd., Tokyo, 1959.

[U] T. UEDA, Critical orbits of holomorphic maps on projective spaces, J. Geom. Anal. 8(2) (1998), 319-334.

[Vi] C. Viterbo, Functors and computations in Floer homology with applcations. I, Geom. Funct. Anal. 9(5) (1999), 985-1033.

[V] A. G. Vitushrin, Some examples related to the problem of polynomial transformations of $\mathbb{C}^{n}$, Math. USSR-Izv. 5 (1971), 269-279.

[We] B. WeICKeRT, Infinite-dimensional complex dynamics: a quantum random walk, Discrete Contin. Dynam. Systems $\mathbf{7 ( 3 )}$ (2001), 517-524.

[W] T. H. WolfF, Plane harmonic measures live on sets of $\sigma$-finite length, Ark. Mat. 31(1) (1993), 137-172. 
John Erik Fornæss:

Department of Mathematics

The University of Michigan

East Hall, Ann Arbor, Mi 48109

U.S.A.

E-mail address: fornaess@math.lsa.umich.edu

Nessim Sibony:

CNRS UMR8628

Department of Mathematics

Université Paris-Sud

Batiment 425

Orsay Cedex

France

E-mail address: Nessim.SIBONY@math.u-psud.fr

Primera versió rebuda el 26 de febrer de 2001, darrera versió rebuda el 14 de març de 2001. 